\title{
Cytogenetic investigations in wheat, rye and triticale. I. Evaluation of improved Giemsa C- and fluorochrome banding techniques in rye chromosomes
}

\author{
J. Martin and \\ C. U. Hesemann
}

\author{
Universität Hohenheim, Institut für Genetik (240), \\ Garbenstraße 30, 7000 Stuttgart 70, \\ Federal Republic of Germany.
}

Giemsa's solution and the fluorescent agents Hoechst 33258, DAPI and quinacrine were applied to chromosomes of rye in order to obtain reliable banding techniques. Methodical improvements led to distinct Giemsa C-banding patterns revealing a high number of interstitial bands which allow the discrimination even between closely related rye forms. Hoechst 33258 performed a clear fluorescent banding pattern in rye chromosomes while DAPI showed a similar but less bright fluorescence. Quinacrine proved to be an inadequate tool for rye cytogenetics, although in interphase nuclei regions of brighter fluorescence appeared. Comparing Giemsa $C$ - and Hoechst 33258 banding, the latter could serve for a rapid identification of rye chromosomes, however, Giemsa C-banding is the method at choice for detailed analysis.

\section{INTRODUCTION}

The development of banding techniques that allow a differential staining of heterochromatic chromosomal segments made it possible to identify individual chromosomes (Caspersson et al., 1968; Pardue and Gall, 1970). Today such methods are indispensable in modern cytogenetics, not only for karyotype analysis, but also in order to detect structural and numerical aberrations of chromosomes. Moreover, banding techniques are helpful for investigations with regard to evolutionary relationships and in order to reveal differences in chromosomal structure between individuals of the same species or population.

Rye plays a prominent role in plant cytogenetics (e.g., Sarma and Natarajan, 1973; Verma and Rees, 1974; Weimarck, 1975; Lelley et al., 1978; Schlegel et al. 1986). Also the increasing impact of hybrid rye and of the interspecific wheatrye bastard triticale requires accurate knowledge about banding patterns of rye materials.

Banding techniques can be divided into two groups according to the stain which is applicated. Giemsa's solution is the most important of a group of nonfluorescent stains. Banding with a second group of stains, the fluorochromes, is commonly employed to a lesser extent. Fluorescent banding methods for rye chromosomes were first published by Sarma and Natarajan (1973) and Vosa (1974) for the bibenzimidazole derivative "Hoechst 33258 " and for quinacrine, however, only the Hoechst technique was successful in differentiating heterochromatic segments. Another interesting fluorochrome is DAPI (4'-6-diamidino-2-phenylindol), which can be successfully applied to stain rye chromosomes (Schlegel et al., 1986).

With respect to the inherent difficulties in reproducing consistent results using existing methods it was our intention to evolve a reliable Giemsa C-banding technique for rye chromosomes which also allows the identification of a large number of C-bands. The second purpose of this work was to develop also a practicable and reproducible fluorescent banding technique by testing the fluorochromes quinacrine, Hoechst 33258 and DAPI.

\section{MATERIALS AND METHODS}

\section{Plant materials}

Rye cultivar "Halo" (Petkus) was taken as a standard for testing the different banding techniques. In addition we used the rye inbred lines L111, L188 and L281 from the Hohenheim selections. All materials were kindly supplied by Prof. $\mathrm{Dr}$ H. H. Geiger, Lehrstuhl für Populationsgenetik, Universität Hohenheim. 


\section{Pretreatment}

Seeds were germinated in petri dishes at $20^{\circ} \mathrm{C}$ in the dark. Roots of $1-2 \mathrm{~cm}$ were collected and treated with 0.02 per cent colchicine on filter paper for about $2 \mathrm{~h}$. After fixation in ethanol/glacial acetic acid $(3: 1)$ at $4^{\circ} \mathrm{C}$ overnight the material was stored in 90 per cent ethanol for up to four weeks.

\section{Preparation of the slides}

The ethanol was washed out by distilled water for $10 \mathrm{~min}$. After a mild hydrolysis in $0.1 \mathrm{~N} \mathrm{HCl}$ at $56^{\circ} \mathrm{C}$ for $4 \mathrm{~min}$ the roots were treated with a solution of 5 per cent pectinase (Rohament P5 from Aspergillus niger, 0.45 units/mg, SERVA, Heidelberg) for 1-2 h. Maceration was sufficient if the tips of the roots bent easily but did not fall off. Successively the roots were rinsed in distilled water for $2 \times 7 \mathrm{~min}$ and incubated in 45 per cent acetic acid. The squashing of the meristematic parts of the root tips $(1-2 \mathrm{~mm})$ is described in detail here because this is indeed the most important step of the procedure: In a very small drop of 45 per cent acetic acid on a slide the tissue is plucked to pieces by a preparation needle and a sharp scalpel. One more drop of liquid is added and the slide heated very gently over an ethanol flame. A cover-glass is fitted over the tightened drop and tapped lightly with a needle until the cells have spread, becoming almost invisible. The excess acetic acid is removed from the edges of the coverglass using blottingpaper. A piece of blotting-paper is layed over the slide and carefully squashed once with the thumb. After freezing on dry ice and removing the coverslips the slides were transferred into absolute ethanol for 3-5 min and dried by air.

\section{Giemsa C-banding}

Slides which had been air-dried for about one week were put into a filtrated barium hydroxide solution (4 per cent), which was freshly prepared and kept in an oven at $50-55^{\circ} \mathrm{C}$. This treatment lasted exactly $10 \mathrm{~min}$. The slides were rinsed thoroughly in running deionized water for $5 \mathrm{~min}$ and incubated in a jar of distilled water for $50 \mathrm{~min}$. After $2 \times$ SSC-treatment $\left(0.33 \mathrm{M}, 1 \mathrm{~h}, 60-65^{\circ} \mathrm{C}, p \mathrm{H} 7\right)$ the slides were again rinsed in distilled water $2 \times 5 \mathrm{~min}$ and put into a staining jar with 3 per cent Giemsa's solution (MERCK, Darmstadt) diluted with Sörensen's buffer $p \mathrm{H} 6.7$. Staining occurs very slowly in about 1-2 days at $20^{\circ} \mathrm{C}$ necessitating periodic monitoring. If staining was satisfactory the slides were rinsed carefully for 10-20 s in distil- led water and mounted in Euparal (CHROMA, Stuttgart) after drying by air for one day.

\section{Hoechst 33258 banding}

For Hoechst banding the air drying of the squash preparations should not exceed 2-3 days. The slides were rehydrated by ethanol steps $(70,50$, 30,0 per cent). Five minutes was enough for each step. After a pre-incubation in McIlvaine's buffer of $p \mathrm{H} 5.5$ for $10 \mathrm{~min}$ staining took place $10-30 \mathrm{~min}$ in $2 \mu \mathrm{g} / \mathrm{ml}$ Hoechst 33258 dissolved in the same buffer. To obtain the desired concentration it was useful to dilute aliquots of a stock solution of the fluorochrome $(100 \mu \mathrm{g} / 10 \mathrm{ml}$ distilled water $)$. The exact staining time had to be evaluated using control preparations for the respective staining series. To remove surplus stain the slides were rinsed in McIlvaine's buffer $p \mathrm{H} 5 \cdot 5$ for $2 \times 1 \mathrm{~min}$ and distilled water $2 \times 2 \mathrm{~min}$, briefly dried by blowing air over the surface of the slides, immediately mounted with 60 per cent sucrose dissolved in distilled water, and kept in a cool place overnight. The coverslips were edged by colourless nail varnish. Preserved in this manner the preparations can be stored in the dark at $4^{\circ} \mathrm{C}$ for more than 6 months until examination under the fluorescence microscope.

\section{DAPI banding}

The method for DAPI was the same as described for Hoechst 33258 with the following exceptions: The ethanol steps were replaced by a $15 \mathrm{~min}$ incubation in distilled water and the concentration of this fluorochrome was $1 \mu \mathrm{g} / \mathrm{ml}$. Staining time was 10-15 min and the durability of the preparations several weeks.

\section{Quinacrine banding}

Air drying after squash preparation had to be strictly avoided. After the slides had been frozen on dry ice to remove the coverslips they were placed into a dish of glacial acetic acid for $20 \mathrm{~s}$ and then transferred into absolute ethanol for $5 \mathrm{~min}$. Staining took place in a solution of 0.25 per cent quinacrine hydrochloride in absolute ethanol during $10-20 \mathrm{~min}$. The slides were washed $3 \times$ $2 \mathrm{~min}$ in 96 per cent ethanol, $2 \times 3 \mathrm{~min}$ in distilled water, and for short-term examination without air drying mounted in distilled water. For long-term observation they could be preserved by mounting in 60 per cent sucrose after quickly blowing away the water from the surface of the slides by com- 
pressed air. By no means should the preparations be allowed to dry up. The preparations were durable for at most 1-2 days.

\section{Fluorescence equipment}

The optical outfit consisted of a ZEISS (Oberkochen) microscope WL provided with an epifluorescence equipment and a mercury light source $\mathrm{HBO} 50 \mathrm{~W}$. The filters are specified in table 1 .

Table 1 Filter combinations for fluorescence studies

\begin{tabular}{lll}
\hline Filters & $\begin{array}{l}\text { For } \\
\text { Hoechst/DAPI }\end{array}$ & For quinacrine \\
\hline Excitation filter & UG 1/3 & BP 433 \\
Beam splitter & FT 420 & FT 460 \\
Barrier filter & LP 418 & LP 470 \\
\hline
\end{tabular}

\section{Photography}

Microphotographs of fluorescent banding were taken on an ILFORD PAN F film, for Giemsa C-banding on AGFAORTHO 25 film, using a ZEISS C35 camera.

\section{RESULTS AND DISCUSSION}

The Giemsa C-banding patterns resulting from the technique described lead to an increased number of interstitial bands (fig. 1). Connections between these and the chromomere pattern of pachytene chromosomes can be implied according to Nagl (1976). Further investigations about early mitotic metaphase and meiotic pachytene stages by means of Giemsa C-banding will give more evidence about this question. In comparison with other exemplary publications since Vosa (1974) a progress in the detected amount of C-bands in the genome can be noticed (table 2). It is our opinion

Table 2 Maximum number of interstitial and telomeric Giemsa C-bands in the haploid genome of rye given in a sample of publications (modified after Hesemann et al., 1986)

\begin{tabular}{ll}
\hline Authors & C-bands \\
\hline Vosa (1974) & 23 \\
Hadlaczky and Koczka (1974) & 25 \\
Giraldez et al. (1979) & 27 \\
Schlegel et al. (1986) & 28 \\
Badaeva et al. (1986) & 29 \\
Semenov and Semenova (1982) & 30 \\
De Vries and Sybenga (1976) & 34 \\
Lelley et al. (1978) & 34 \\
Hesemann et al. $(1987)$ & 59 \\
\hline
\end{tabular}

that this development has still not reached its end, so that for all we know the detection of more than $80 \mathrm{C}$-bands in the haploid rye genome seems to be possible. However, an achievement of a uniform Giemsa C-banding method, hitherto different in any publication, should be taken into account.

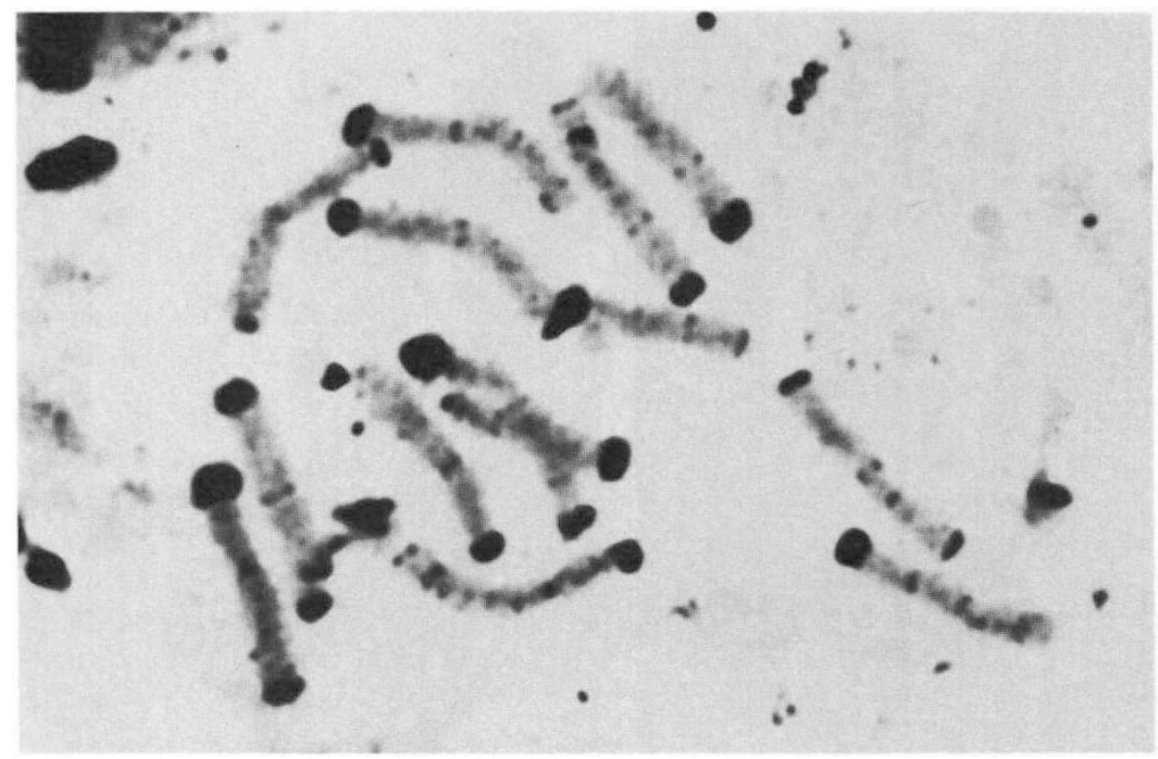

Figure 1 Giemsa C-banding in a metaphase cell of L281. 
With the co-operation of all rye cytologists, who are interested, such a standard technique may be established on the basis of the presented method. In this context we also welcome the standard band nomenclature for rye chromosomes proposed by Schlegel et al. (1986).

During our experiments to establish a reproducible C-banding method we observed the following steps of the procedure to be of great relevance to success or failure:

-During $\mathrm{HCl}$-hydrolysis, it is essential to strictly observe the appointed temperature given for an alcohol thermometer. Too low temperature results in an insufficient clearing of the cytoplasm, too high temperature results in complete failure of the banding.

-Enzymatic maceration must be adapted to the specific charges of pectinase delivered by the producer. The enzyme should be washed out carefully, if not, banding will be inhibited.

- Squash preparation causes the most deviations of all. Leaving all other steps constant and only changing the persons doing the squash preparation often leads to more different results than varying any other step.
- Air drying of the slides after squash preparation is necessary for at least five days. A longer period of maturation will shorten the staining time.

-Denaturation with barium hydroxide is also a delicate step and should be carried out according to the instructions given.

Differentiation even between closely related forms is possible with this Giemsa C-banding technique. For example between cv. "Halo" and the inbred line L188 obvious differences in the banding patterns can be observed, although both belong to the same source population. The chromosomes are presented in fig. 2. Due to homozygosity inbred lines show a more constant banding pattern, although a lower amount of interstitial bands in total, but the C-bands of the heterozygous cultivar "Halo" often seem more inconsistent. These observations correspond well with our results about the polymorphism of rye chromosomes (Hesemann et al., 1987).

Application of the Hoechst 33258 banding technique resulted in specific fluorescent patterns at heterochromatic regions of rye chromosomes (fig. 3). According to our experience fluorochrome banding with Hoechst 33258 is not as susceptible

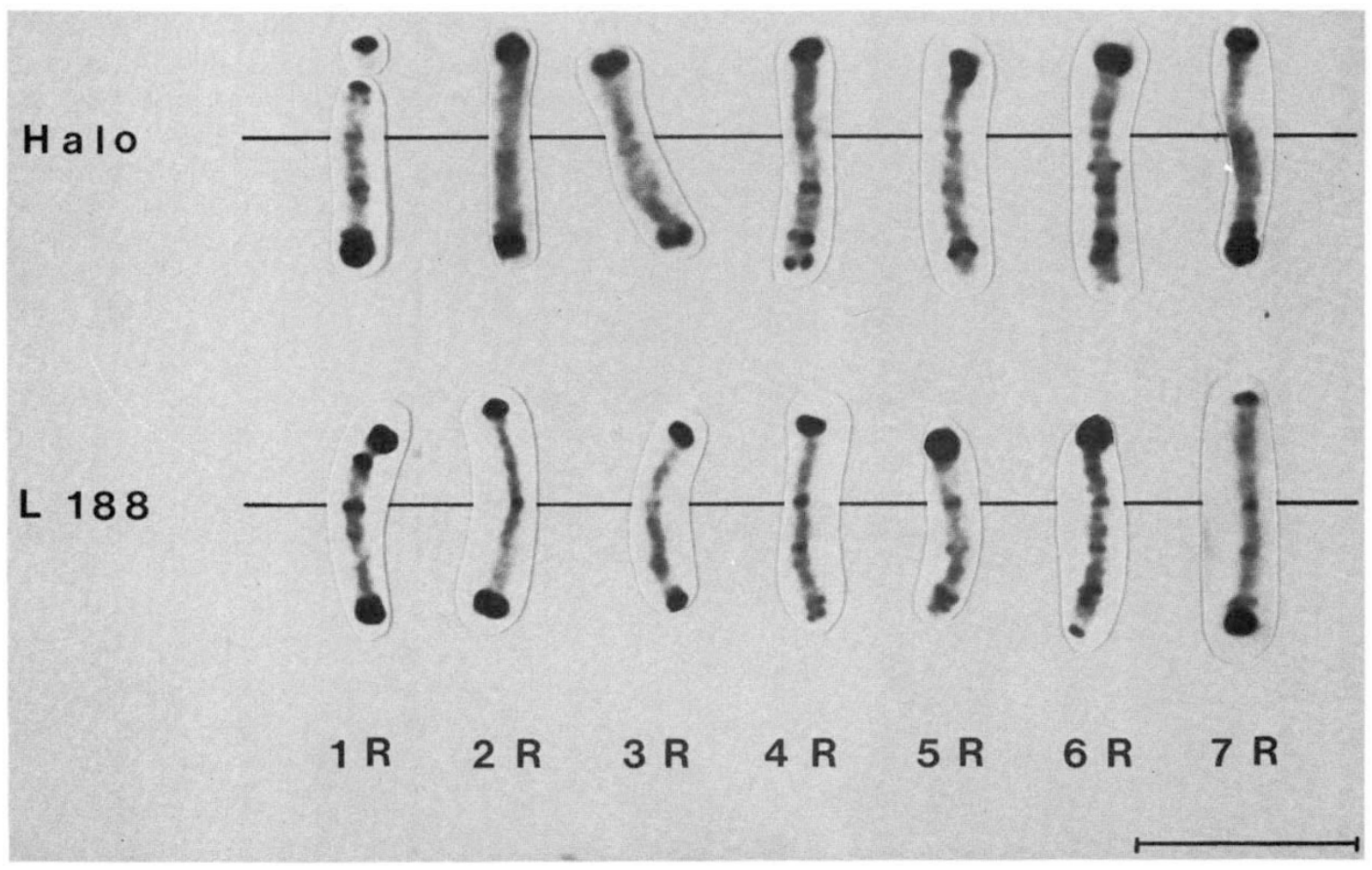

Figure 2 Giemsa C-banded chromosomes of cv. "Halo" and inbred line L188. Bar =10 $\mu \mathrm{m}$. 


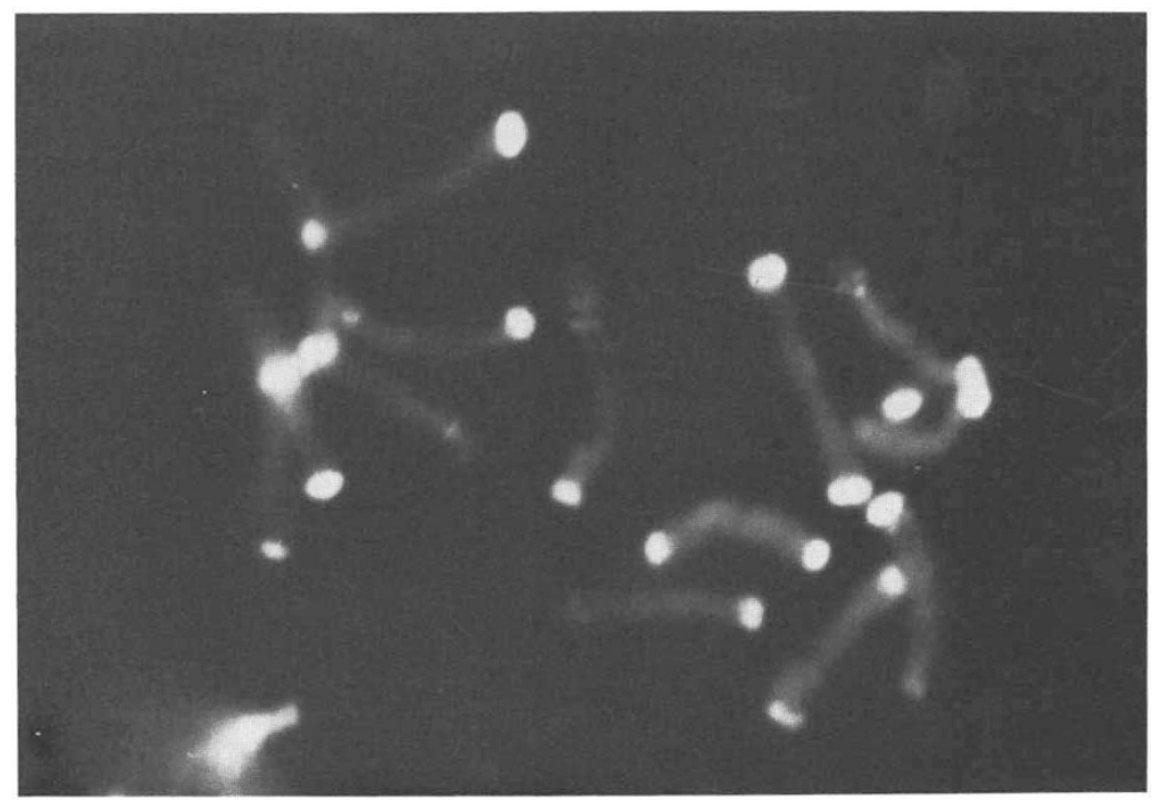

Figure 3 Hoechst 33258 banding in a metaphase cell of cv. "Halo".

to methodical deviations as Giemsa C-banding. The decisive factors of the technique described are as follows:

- $\mathrm{HCl}$-hydrolysis should not reach $60^{\circ} \mathrm{C}$ in temperature, otherwise this would give rise to unsatisfactory banding results. With temperatures of about $60^{\circ} \mathrm{C}$ the nucleoli will be seen as "dark holes" in the interphase nuclei. Similar observations have been described by Laloue et al. (1980) for nuclei of Nicotiana and Asparagus.

- Rehydratisation of the slides proved to be necessary. For rye chromosomes Sarma and Natarajan (1973) put the air-dried slides directly into the staining solution. This was not successful in our own experiments. Improved results were achieved by using a rehydratisation with ethanol steps and a pre-incubation in distilled water and McIlvaine's buffer (Caspersson et al., 1970; Schweizer and Nagl, 1976).

-Banding techniques with Hoechst 33258 differ widely in staining time, concentration of the stain, solution medium and its $p \mathrm{H}$. In our test series for $p \mathrm{H} 4.0$ up to $p \mathrm{H} 7.0$ the brightest fluorescence was revealed at $p \mathrm{H} \mathrm{5.5.} \mathrm{At} p \mathrm{H} 7$ the fluorochrome is unfortunately bound to the cell walls changing into a faint yellow tone at $p \mathrm{H} 4$.

-Washing out the surplus stain is necessary to inhibit background fluorescence. On the other hand the rinsing should not last longer than
$6 \mathrm{~min}$, otherwise the slides will de-stain and the fading effect will be strengthened.

-The most critical point of fluorescent banding is the choosing of the appropriate mounting medium. Vosa (1974) used distilled water and glycerine mixtures, Das et al. (1979) mounted the slides in Sörensen's buffer or in a glycerol buffer mixture. These mediums are sufficient for short-term examination of a few slides. For the investigation of more slides a way of mounting is required which enables preparations of a longer durability. We found moisture chambers not useful for this purpose. The best mounting medium proved to be 60 per cent sucrose. In opposition to Lee and Collins (1977), who dissolved the sugar in McIlvaine's buffer for their Q-banding experiments, we detected that for Hoechst 33258 a solution in distilled water brings about the best vision under the fluorescence microscope.

- The slides should be irradiated only after a period of cool storage in the dark for at least one night or longer because immediate examination gives rise to an intensified fading effect.

The Hoechst 33258 banding technique resulted in two types of fluorescent bands. Telomeric bands revealed a strong and bright fluorescence and interstitial bands showed a faint but distinct fluorescence for a few characteristic bands of the rye 


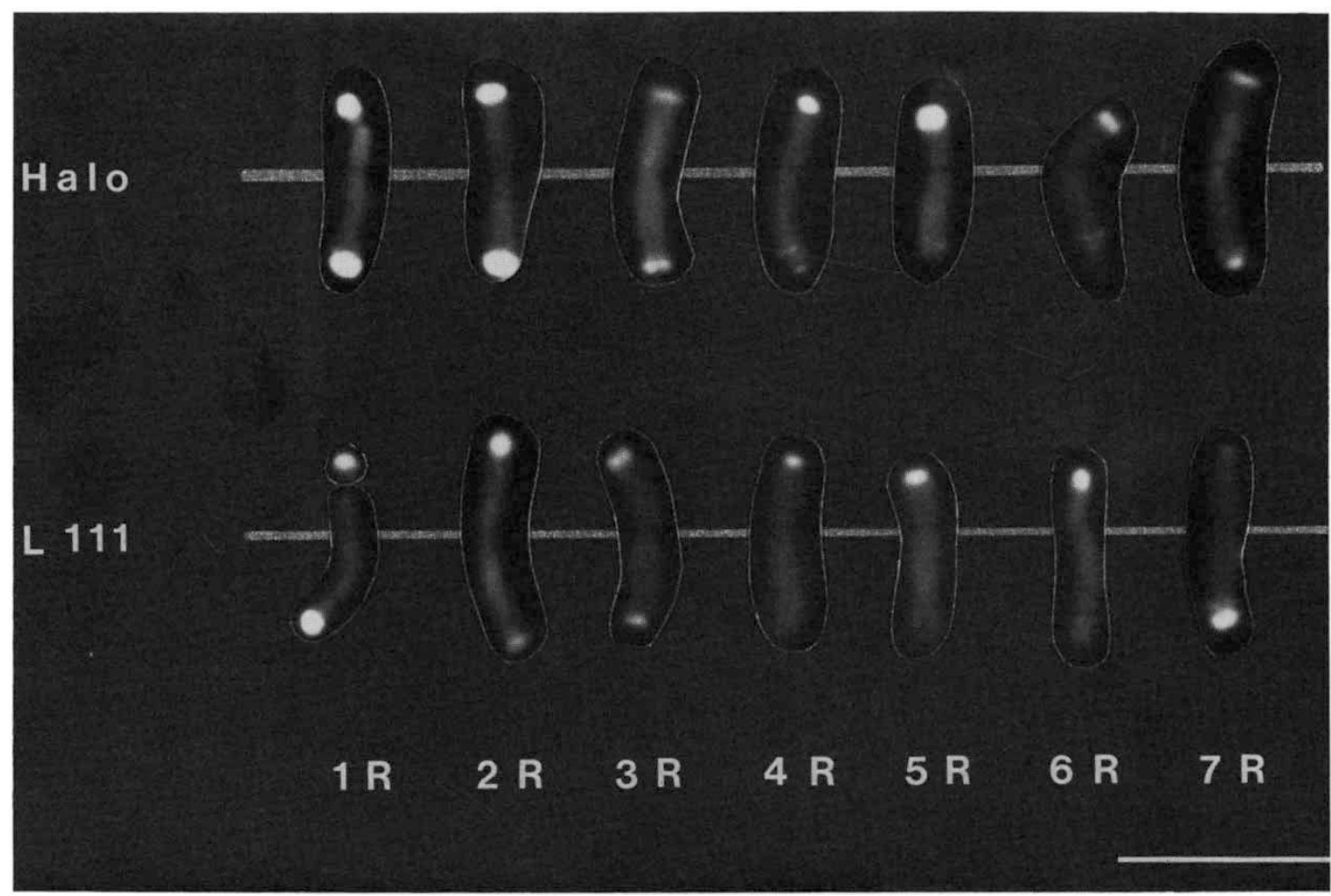

Figure 4 Hoechst 33258 banded chromosomes of cv. "Halo" and inbred line L111. Bar $=10 \mu \mathrm{m}$.

genome. In the chromosomes of Halo and L111 after Hoechst staining (fig. 4) only rough differences are visible particularly with regard to the extent of telomeric bands. Discriminating different rye forms by this type of banding will be difficult, if no fundamental differences in the distribution or amount of telomeric heterochromatin are present. As for Halo and L111, although closely related, the chromosomes $1 \mathrm{R}, 4 \mathrm{R}, 5 \mathrm{R}$ and $7 \mathrm{R}$ could be discerned not only by telomeric but to some extent also by interstitial bands.

For Hoechst 33258 banding Sarma and Natarajan (1973) gave a nomenclature for five different types of chromosomes which can be allied to the standard nomenclature as shown in table 3 . The same authors complained that interstitial bands had been found only in early metaphase stages. This cannot be confirmed from our results.

DAPI-banding of rye chromosomes is reported by Schlegel et al. (1986) from unpublished results. In our own experiments DAPI showed similar banding patterns as Hoechst 33258 but photodocumentation was embarassed by less bright fluorescence.
Table 3 Five types of Hoechst 33258 banded rye chromosomes as described by Sarma and Natarajan (1973) allied to the standard nomenclature (Sybenga, 1983)

\begin{tabular}{ll}
\hline Sarma and Natarajan & Standard nomenclature \\
\hline Type A & $2 \mathrm{R} / 3 \mathrm{R}$ \\
Type B & $6 \mathrm{R}$ \\
Type C & $1 \mathrm{R}$ \\
Type D & $7 \mathrm{R}$ \\
Type E & $4 \mathrm{R} / 5 \mathrm{R}$ \\
\hline
\end{tabular}

Attempts of a Q-banding method for rye chromosomes were described by Sarma and Natarajan (1973) and Vosa (1974). They failed to establish a Q-banding pattern. Also our own test series were not successful in achieving a clear banding pattern in metaphase chromosomes, however, in interphase kernels (fig. 5(d)) and partially in prophase chromosomes areas of brighter fluorescence appeared. So Q-banding of rye chromosomes does not seem impossible in future if the following critical points are investigated further: 

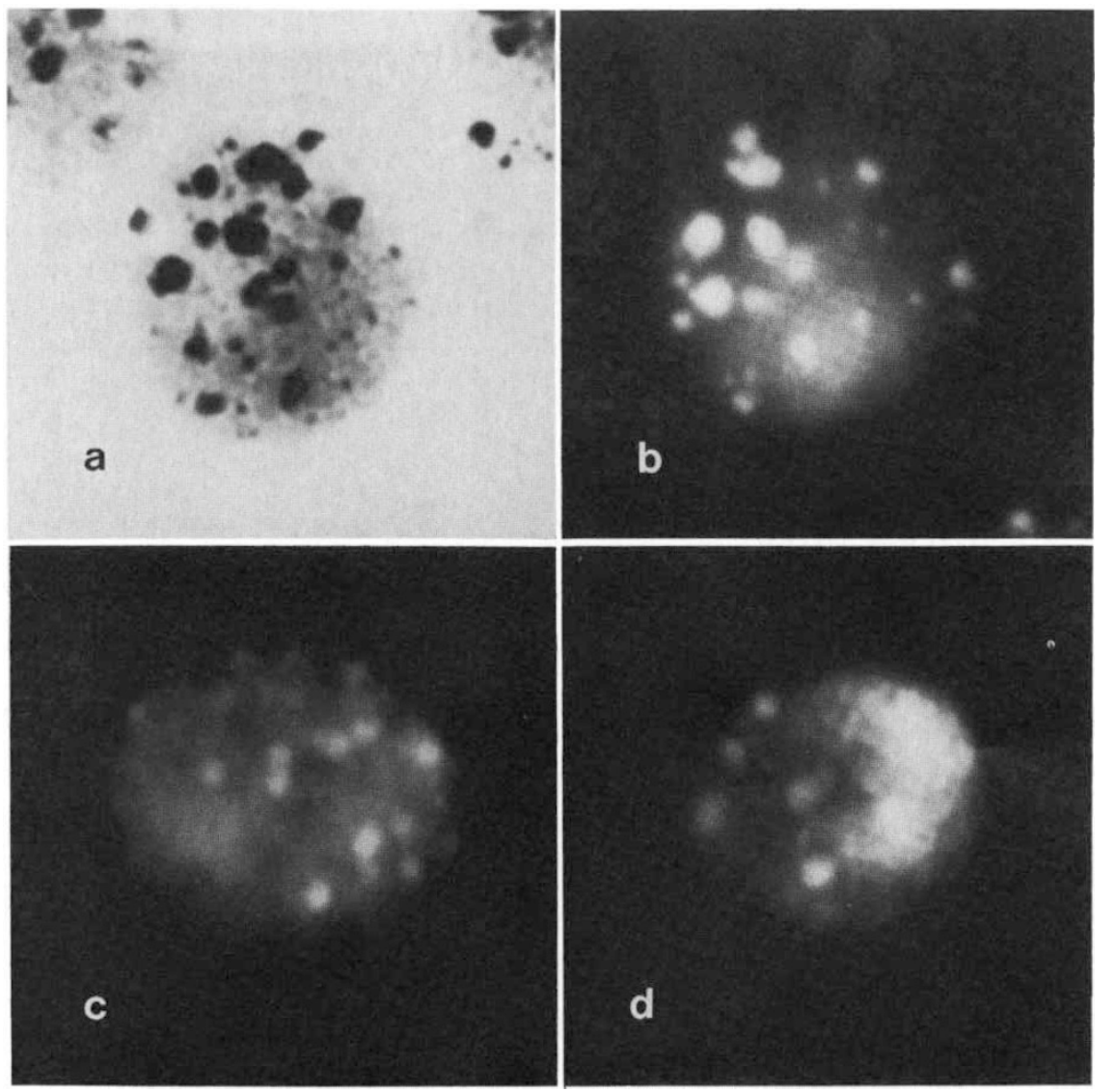

Figure 5 Interphase cell nuclei of cv. "Halo" stained with Giemsa (a), Hoechst 33258 (b), DAPI (c) and quinacrine (d).

- Variation of the $\mathrm{HCl}$-hydrolysis did not affect the results of the quinacrine staining. Kongsuwan and Smyth (1977) replaced this step by a long maceration in 45 per cent acetic acid and attained a Q-banding pattern of Lilium chromosomes. They also observed chromosomes surrounded by cytoplasm not being stained differentially since single chromosomes spread outside from the cells revealed a bright Qbanding pattern. However, using the above method we did not observe any banding in rye chromosomes.

-Cellplasm has the negative character to bind the stain, so that background fluorescence will interfere with the possibility of observation. In order to reduce this effect we used a short treatment with glacial acetic acid directly after removing the coverslips.

-Unquestionably the dispense with air drying before and after staining seems to be of advantage.
In comparison of Giemsa and fluorochrome staining of rye cell nuclei the superiority of Giemsa C-banding over all the three fluorochromes is clearly illustrated in fig. 6 for chromosome 1R. As a result of a comparison between Giemsa C- and Hoechst 33258 banding the following evidence can be given:

- Giemsa C-banding leads to a much higher number of bands than Hoechst fluorescent banding, particularly interstitial bands are reduced, which is in part due to the physical and technical limits of fluorescent microscopy.

-Centromeric heterochromatin does not appear as a band with Hoechst 33258 . The positions of the centromeres, however, are easily discernable by the constrictions of the chromosomes.

-Without regard to the lower number of bands the Hoechst 33258 banding pattern corresponds well with Giemsa C-bands. Hoechst bands were detected at only the same regions where usually distinct Giemsa C-bands had been observed. 


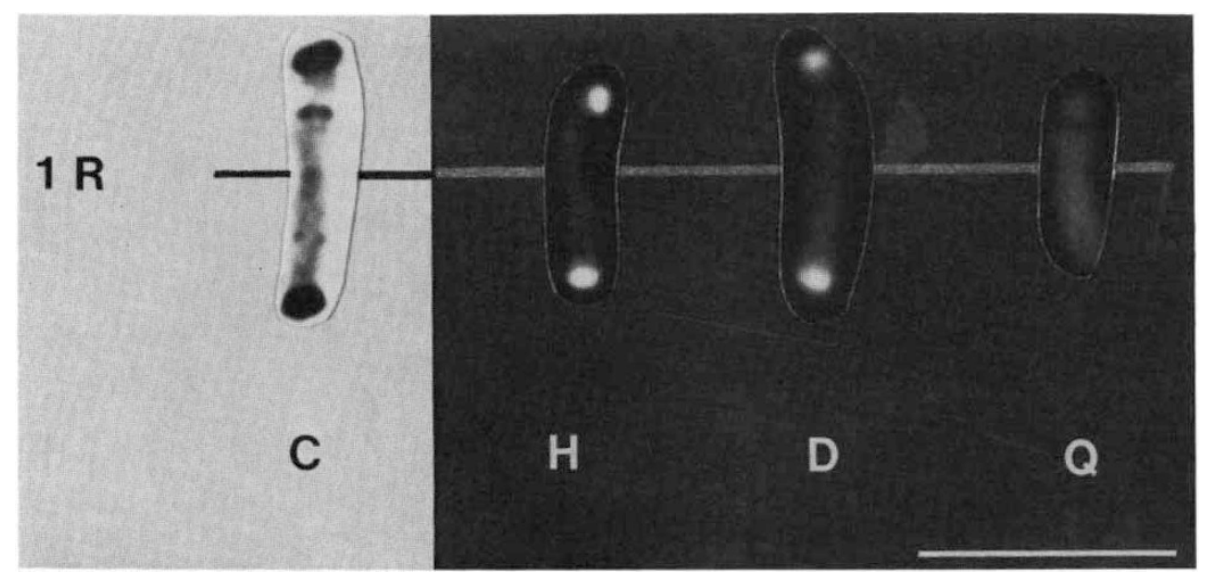

Figure 6 Comparison of Giemsa- and fluorescent staining (Giemsa $=$ C, Hoechst $=H$, DAPI $=$ D and quinacrine $=Q)$ exemplified by somatic chromosome $1 R$. Bar $=10 \mu \mathrm{m}$.

- Two interstitial Giemsa C-bands of the same intensity must not have an analogy in Hoechst banding, so that one or the other band can appear fluorescent possibly due to different types of heterochromatin.

-Telomeric bands in common are comparable with regard to both methods.

In addition to banding patterns of metaphase chromosomes comparison of interphase cell nuclei is of interest to reveal differences between the investigated stains. Figs 5(a) and (b) show that the main chromocentres correspond well between Giemsa C- and Hoechst 33258 banding. DAPI displays a lower bright fluorescence and the chromocentres are not as clearly visible (fig. 5(c)). Quinacrine staining exhibits the lowest number of chromocentres and the nuclei appear more condensed (fig. 5(d)). It would be of interest to know at which sides in metaphase chromosomes these fluorescent regions are located. As mentioned above, it was not yet possible to answer this question.

The described Giemsa technique provides a wide range of interstitial bands, so that just slight differences in chromosomal structure become visible. So far it is the appropriate method for investigating problems of evolutionary or chromosomal importance in detail.

Concerning the fluorochromes, only Hoechst 33258 is recommended yet as a practical tool for rye cytogenetics. It is still not being used far beyond experimental research, however, it could be applicated for example, as a fast method to identify the number of rye chromosomes in wheat-rye substitution or addition lines and in triticale. With regard to this crop, it is also desirable to screen a large number of rye forms in order to evaluate the extent of telomeric heterochromatin. In this manner a contribution could be made to clarify the role of telomeric heterochromatin in triticale cytogenetics.

To sum up, Giemsa C-banding without doubt has the main significance up to now, but at least fluorochrome banding with Hoechst 33258 will be of practical use for special purposes.

Acknowledgements This work has been supported by the Vater und Sohn Eiselen-Stiftung in Ulm.

\section{REFERENCES}

BADAEVA, E. D., BADAEV, N. S., BOLSheVA, N. L. AND ZELININ, A. V. 1986. Chromosome alterations in the karyotype of triticale in comparison with the parental forms. I. Heterochromatic regions of $\mathrm{R}$ genome chromosomes. Theor. Appl. Genet., 72, 518-523.

CASPERSSON, T., FARBER, S., FOLEY, G. E., KUDYNOWSKI, J., MODEST, E. J., SIMONSSON, E., WAGH, U. AND ZECH, L. 1968. Chemical differentiation along metaphase chromosomes. Exp. Cell Res., 49, 219-222.

CASPERSSON, T., ZECH, L., JOHANSSON, C. AND MODEST, E. J. 1970. Identification of human chromosomes by DNAbinding fluorescent agents. Chromosoma, 30, 215-227.

DAS, B. C., RAMAN, R. AND SHARMA, T. 1979. Chromosome condensation and Hoechst fluorescence in meiotic chromosomes of the grasshopper Spathosternum prasiniferum (Walker). Chromosoma, 70, 251-258.

GIRALDEZ, R., CERMEÑO, M. C. AND ORELLANÄ, J. 1979. Comparison of C-banding pattern in the chromosomes of inbred lines and open pollinated varieties of rye. Z. Pflanzenzüchtg., 83, 40-48.

HADLACZKY, GY. AND KOCZKA, K. 1974. C-banding karyotype of rye from hexaploid triticale. Cereal Res. Com., Vol. 2, No. 4, 193-197.

HESEMANN, C. U., PFEIFFER-SCHAD, B. AND MARTIN, J. 1987. Cytogenetic investigations in wheat, rye and triticale. II. 
Extent of heterochromatin polymorphism in cultivars and inbred lines of rye (Secale cereale L.), Plant breeding, 98, 297-305.

HESEMANN, C. U., PFEIFFER, B., MARTIN, J. AND HAINER, A. 1986. Cytogenetic and cytochemical investigations in primary triticale and its parents. Proc. Internat. Triticale Symposium, Sydney, pp. 336-339.

KONGSUWAN, K. AND SMYTH, D. R. 1977. Q-bands in Lilium and their relationship to C-banded heterochromatin. Chromosoma, 60, 169-178.

LALOUE, M., COURTOIS, D. AND MANIGAUlt, P. 1980. Convenient and rapid fluorescent staining of plant cell nuclei with '33258' Hoechst. Plant Sci. letters, 17, 175-179.

LEE, C. S. AND COLliNS, L. 1977. Q- and C-bands in the metaphase chromosomes of Drosophila nasutoides. Chromosoma, 61, 57-60.

Lelley, T., JOSIFEK, K. AND KAltSikes, P. J. 1978. Polymorphism in the Giemsa C-banding pattern of rye chromosomes. Can. J. Genet. Cytol., 20, 307-312.

NAGL, w. 1976. Zellkern und Zellzyklen. Ulmer Verlag, Stuttgart. PARDUE, M. L. AND GALL, J. G. 1970. Chromosomal localisation of mouse satellite DNA. Science, 168, 1356-1358.

SARMA, N. P. AND NATARAJAN, T. 1973. Identification of heterochromatic regions in the chromosomes of rye. Hereditas, 74, 233-238.
SCHLEGEL, R., MELZ, G. AND METTIN, D. 1986. Rye cytology, cytogenetics and genetics-Current status. Theor. Appl. Genet., 72, 721-734.

SCHWEIZER, D. AND NAGL, w. 1976. Heterochromatin diversity in Cymbidium, and its relationship to differential DNA replication. Exp. Cell Res, 98, 411-423.

SEMENOV, V. I. AND SEMENOVA, E. V. 1982. Polymorphism of the rye chromosomes for the heterochromatin bands in some rye varieties and triticales. Genetika, Vol. 18, No. 11, 1856-1867.

SYBENGA, J. 1983. Rye chromosome nomenclature and homoeology relationships/Workshop report. Z. Pflanzenzüchtg., 90, 297-304.

VERMA, S. C. AND REES, H. 1974. Giemsa staining and the distribution of heterochromatin in rye chromosomes. Heredity, 32, 118-122.

DE VRIES, J. N. AND SYBENGA, J. 1976. Identification of rye chromosomes: The Giemsa banding pattern and the translocation tester set. Theor. Appl. Genet., 48, 35-43.

VOSA, C. G. 1974. The basic karyotype of rye (Secale cereale L.) analysed with Giemsa and fluorescence methods. Heredity, 33, 403-408.

WEIMARCK, A. 1975. Heterochromatin polymorphism in the rye karyotype as detected by the Giemsa C-banding technique. Hereditas, 79, 293-300. 